\title{
Second order linear recursions whose subscripts are a power
}

\author{
Emrah Kilic and Elif Tan
}




\title{
SECOND ORDER LINEAR RECURSIONS WHOSE SUBSCRIPTS
} ARE A POWER

\author{
EMRAH KILIC AND ELIF TAN
}

Received 3 March, 2010

\begin{abstract}
We consider two kinds of second order linear recurrences whose subscripts are powers and present certain new identities including these recurrences. Furthermore, we derive first-order nonlinear homogeneous recurrence relations for these recurrences. Our results generalize earlier results as well as they provide new solutions for certain uncompleted cases of the literature.
\end{abstract}

2000 Mathematics Subject Classification: 11B37

Keywords: Fibonacci and Lucas number, recurrence relation, polynomial

\section{INTRODUCTION}

Let $p$ be a nonzero integer such that $\Delta=p^{2}+4 \neq 0$. Define the generalized Fibonacci type $\left\{u_{n}\right\}$ and Lucas type $\left\{v_{n}\right\}$ sequences as follows:

$$
u_{n}=p u_{n-1}+u_{n-2}
$$

and

$$
v_{n}=p v_{n-1}+v_{n-2},
$$

where $u_{0}=0, u_{1}=1$ and $v_{0}=2, v_{1}=p$, respectively.

If the roots of $x^{2}-p x-1=0$ are $\alpha$ and $\beta$, then the Binet forms of $\left\{u_{n}\right\}$ and $\left\{v_{n}\right\}$ are

$$
u_{n}=\frac{\alpha^{n}-\beta^{n}}{\alpha-\beta} \text { and } v_{n}=\alpha^{n}+\beta^{n} .
$$

If $p=1$, then $u_{n}=F_{n}$ ( $n$th Fibonacci number) and $v_{n}=L_{n}$ ( $n$th Lucas number) respectively.

Usiskin $[7,8]$ suggested the following problems: For $n>0$, show that

$$
F_{3^{n}}=\prod_{k=0}^{n-1} L_{2.3^{k}}-1
$$

and

$$
L_{3^{n}}=\prod_{k=0}^{n-1} L_{2.3^{k}}+1
$$


In [4], the author asked for the solution of the first order cubic recurrence relation:

$$
a_{n+1}=5 a_{n}^{3}-3 a_{n}
$$

with $a_{0}=1$.

Then in [6], the solution is given as $a_{n}=F_{3^{n}}$. The same problem appeared as Problem 1809 in Crux Mathematicorum 20 (1994): 19-20.

In the same issue, there was a proposal to solve the recurrence

$$
P_{n+1}=25 P_{n}^{5}-25 P_{n}^{3}+5 P_{n}, P_{0}=1 .
$$

The solution was given as $P_{n}=5^{n}$. Also the following recurrences and their solutions were commented by Murray S. Klamkin as an addendum to the solution of the problem given in [6]:

$$
\begin{aligned}
& A_{n+1}=A_{n}^{2}-2, A_{1}=3, \\
& B_{n+1}=B_{n}^{4}-4 B_{n}^{2}+2, \quad B_{1}=7, \\
& C_{n+1}=C_{n}^{6}-6 C_{n}^{4}+9 C_{n}^{2}-2, C_{1}=18,
\end{aligned}
$$

where $A_{n}=L_{2^{n}}, B_{n}=L_{4^{n}}$ and $C_{n}=L_{6^{n}}$.

In [1], the author presented some identities involving Fibonacci numbers of the form $F_{k^{n}}$ for positive odd $k$ and gave a first-order nonlinear homogeneous recurrence relation for $F_{k^{n}}$, which generalized (1.3), (1.1) and (1.2).

Recently in [2], Helmut Prodinger gave a general expansion formula for a sum of powers of Fibonacci numbers, as considered by Melham, as well as some extensions.

In this paper, we consider two generalized second order recursion sequences and then generalize the results of [1] for the odd $k$ case as well as derive a new first-order nonlinear homogeneous recurrence relation for the sequence $\left\{u_{k^{n}}\right\}$ for possible even $k$. Further we present that the generalized Lucas numbers $v_{k^{n+1}}$ is a polynomial of generalized Fibonacci numbers $u_{k^{n}}$ of degree $k$ for even $k$.

\section{MAin Results}

In this section, in order to derive a recurrence relation for both even and odd subscripted terms $u_{k^{n}}$, we start with the following result.

Proposition 1. For $n \geq 1$ and even $k$,

$$
u_{k^{n}}=u_{k} \prod_{i=1}^{n-1}\left(\sum_{j=1}^{k / 2} v_{(2 j-1) k^{i}}\right. \text {. }
$$

Proof. Consider

$$
u_{k^{n}}=u_{k} \frac{u_{k^{2}}}{u_{k}} \frac{u_{k^{3}}}{u_{k^{2}}} \ldots \frac{u_{k^{n}}}{u_{k^{n-1}}}=u_{k} \prod_{i=1}^{n-1} \frac{u_{k^{i+1}}}{u_{k^{i}}} .
$$


From [5], we have that for even $k \geq 2$,

$$
\frac{m^{k}-n^{k}}{m-k}=\sum_{j=0}^{k / 2-1}(m n)^{i}\left(m^{k-2 i-1}+n^{k-2 i-1}\right)
$$

When $m=\alpha^{n}, n=\beta^{n}$ in (2.2), we get

$$
\begin{gathered}
\frac{u_{k n}}{u_{n}}=\sum_{j=0}^{k / 2-1}(\alpha \beta)^{j n}\left(\alpha^{(k-2 j-1) n}+\beta^{(k-2 j-1) n}\right)=\sum_{j=0}^{k / 2-1}(-1)^{j n} v_{(k-2 j-1) n} \\
\frac{u_{k^{i}+1}}{u_{k^{i}}}=\sum_{j=0}^{k / 2-1}(-1)^{j k^{i}} v_{(k-2 j-1) k^{i}}=\sum_{j=1}^{k / 2} v_{(2 j-1) k^{i}}
\end{gathered}
$$

By (2.3), equation (2.1) equals to

$$
u_{k^{n}}=u_{k} \prod_{i=1}^{n-1} \frac{u_{k^{i+1}}}{u_{k^{i}}}=u_{k} \prod_{i=1}^{n-1}\left(\sum_{j=1}^{k / 2} v_{(2 j-1) k^{i}}\right) .
$$

Thus the proof is complete.

For the Fibonacci and Lucas case when $p=1$, we also refer to [9].

For later use, we give the following two identities:

$$
\begin{aligned}
\sum_{i=1}^{r}(-1)^{i} v_{a i} & =\sum_{i=1}^{r}(-1)^{i} \alpha^{a i}+\sum_{i=1}^{r}(-1)^{i} \beta^{a i} \\
& =\frac{-\alpha^{a}+(-1)^{r}\left(\alpha^{a}\right)^{r+1}}{1+\alpha^{a}}+\frac{-\beta^{a}+(-1)^{r}\left(\beta^{a}\right)^{r+1}}{1+\beta^{a}} \\
& =\frac{(-1)^{a+r} v_{a r}+(-1)^{r} v_{a(r+1)}-v_{a}-2(-1)^{a}}{v_{a}+1+(-1)^{a}}
\end{aligned}
$$

and

$$
v_{a+b}-(-1)^{b} v_{a-b}=\Delta u_{a} u_{b} .
$$

Proposition 2. For odd $k$ and $n \geq 1$,

$$
u_{k^{n}}=(-1)^{(n-1)(k-1) / 2} u_{k} \prod_{i=1}^{n-1}\left(1+\sum_{j=1}^{(k-1) / 2}(-1)^{j} v_{2 k^{i} j}\right) \text {. }
$$

Proof. If $(k-1) / 2$ is even, we write by (2.4) and (2.5)

$$
u_{k^{n}}=u_{k} \prod_{i=1}^{n-1} \frac{u_{k^{i+1}}}{u_{k^{i}}}=u_{k} \prod_{i=1}^{n-1}\left(1+\frac{\Delta u_{k^{i+1}} u_{k^{i}}}{\Delta u_{k^{i}} u_{k^{i}}}-1\right)
$$




$$
\begin{aligned}
& =u_{k} \prod_{i=1}^{n-1}\left(1+\frac{v_{k^{i+1}+k^{i}}-(-1)^{k^{i}} v_{k^{i+1}-k^{i}}}{v_{k^{i}+k^{i}}-(-1)^{k^{i}} v_{k^{i}-k^{i}}}-1\right) \\
& =u_{k} \prod_{i=1}^{n-1}\left(1+\frac{v_{2 k^{i} \frac{k+1}{2}}+v_{2 k^{i} \frac{k-1}{2}}-v_{2 k^{i}}-2}{v_{2 k^{i}}+2}\right) \\
& =u_{k} \prod_{i=1}^{n-1}\left(1+\sum_{j=1}^{(k-1) / 2}(-1)^{j} v_{2 k^{i} j}\right) \\
& =(-1)^{(n-1)(k-1) / 2} u_{k} \prod_{i=1}^{n-1}\left(1+\sum_{j=1}^{(k-1) / 2}(-1)^{j} v_{2 k^{i} j}\right) .
\end{aligned}
$$

If $(k-1) / 2$ is odd, then we write

$$
\begin{aligned}
u_{k^{n}} & =(-1)^{n-1} u_{k} \prod_{i=1}^{n-1} \frac{u_{k^{i+1}}}{u_{k^{i}}}=(-1)^{n-1} u_{k} \prod_{i=1}^{n-1}\left(1-\frac{\Delta u_{k^{i+1}} u_{k^{i}}}{\Delta u_{k^{i}}}-1\right) \\
& =(-1)^{n-1} u_{k} \prod_{i=1}^{n-1}\left(1+\frac{-v_{2 k^{i} \frac{k+1}{2}}-v_{2 k^{i} \frac{k-1}{2}-v_{2 k^{i}}-2}}{v_{2 k^{i}}+2}\right) \\
& =(-1)^{n-1} u_{k} \prod_{i=1}^{n-1}\left(1+\sum_{k=1}^{(k-1) / 2}(-1)^{j} v_{2 k^{i} j}\right)
\end{aligned}
$$

So we have proved the conclusion for all cases.

\section{RECURRENCE RELATION FOR $\left\{u_{k^{n}}\right\}$}

We shall derive recurrence relations for $\left\{u_{k^{n}}\right\}$ or $\left\{v_{k^{n}}\right\}$ for odd or even $k$. Thus we need the following result:

Lemma 1. For $n, q \geq 0$,

$$
\begin{aligned}
u_{(2 q+1) n} & =u_{n} \sum_{k=0}^{q}(-1)^{n(q+k)} \frac{2 q+1}{q+k+1}\left(p^{2}+4\right)^{k}\left(\begin{array}{c}
q+k+1 \\
2 k+1
\end{array}\right) u_{n}^{2 k}, \\
v_{2 q n} & =\sum_{k=0}^{q}(-1)^{n(q+k)} \frac{2 q}{q+k}\left(\begin{array}{c}
q+k \\
2 k
\end{array}\right)\left(p^{2}+4\right)^{k} u_{n}^{2 k}, \\
v_{(2 q+1) n} & =v_{n} \sum_{k=0}^{q}(-1)^{(n+1)(q+k)} \frac{2 q+1}{q+k+1}\left(\begin{array}{c}
q+k+1 \\
2 k+1
\end{array}\right) v_{n}^{2 k},
\end{aligned}
$$




$$
v_{2 q n}=\sum_{k=0}^{q}(-1)^{(n+1)(q+k)} \frac{2 q}{q+k}\left(\begin{array}{c}
q+k \\
2 k
\end{array}\right) v_{n}^{2 k} .
$$

Proof. The proof can be easily obtained from [5] by considering the classical binomial expansions for $a^{n}-b^{n}$ and $a^{n}+b^{n}$ where $a$ and $b$ are real numbers.

We give a recurrence relation for the sequence $\left\{u_{k^{n}}\right\}$ for odd $k$.

Proposition 3. For odd $k>0$ and $n \geq 0$,

$$
u_{k^{n+1}}=\Delta^{(k-1) / 2} u_{k^{n}}^{k}-\sum_{i=0}^{(k-3) / 2} \triangle^{i} C_{i, k} u_{k^{n}}^{2 i+1}
$$

where the coefficients $C_{i, k}$ are given by

$$
C_{i, k}=(-1)^{(k+1) / 2+i} \frac{2 k}{k+2 i+1}\left(\begin{array}{c}
(k+1) / 2+i \\
2 i+1
\end{array}\right)
$$

for $0 \leq i \leq(k-3) / 2$

Proof. Consider

$$
\begin{aligned}
u_{k^{n}}^{k} & =\frac{1}{\triangle^{k / 2}} \sum_{j=0}^{k}\left(\begin{array}{l}
k \\
j
\end{array}\right)(-1)^{j} \beta^{j k^{n}} \alpha^{(k-j) k^{n}} \\
& =\frac{1}{\triangle^{(k-1) / 2}}\left(u_{k^{n+1}}+\sum_{j=1}^{(k-1) / 2}\left(\begin{array}{l}
k \\
j
\end{array}\right) u_{(k-2 j) k^{n}}\right),
\end{aligned}
$$

where $\Delta$ is defined as before. By (3.1), we obtain for odd $k$,

$$
u_{k^{n+1}}=\triangle^{(k-1) / 2} u_{k^{n}}^{k}-\sum_{j=1}^{(k-1) / 2}\left(\begin{array}{l}
k \\
j
\end{array}\right) u_{(k-2 j) k^{n}} .
$$

Then by (3.2) and Lemma 1, we write

$$
\begin{aligned}
u_{k^{n+1}}= & \Delta^{(k-1) / 2} u_{k^{n}}^{k} \\
& -\sum_{j=1}^{\frac{k-1}{2}} \sum_{i=0}^{\frac{k-1}{2}-j}(-1)^{\frac{k-1}{2}+i-j} \triangle^{i}\left(\begin{array}{c}
k \\
j
\end{array}\right)\left(\begin{array}{c}
\frac{k+1}{2}+i-j \\
2 i+1
\end{array}\right) \frac{k-2 j}{\frac{k+1}{2}+i-j} u_{k^{n}}^{2 i+1}
\end{aligned}
$$

which, after reversing the order of summation, can be rewritten as

$$
u_{k^{n+1}}=\triangle^{(k-1) / 2} u_{k^{n}}^{k}-\sum_{i=0}^{(k-1) / 2} \triangle^{i} A_{i, k} u_{k^{n}}^{2 i+1},
$$


where

$$
A_{i, k}=\sum_{j=1}^{(k-1) / 2-i}(-1)^{(k-1) / 2+i-j}\left(\begin{array}{l}
k \\
j
\end{array}\right)\left(\begin{array}{c}
(k+1) / 2+i-j \\
2 i+1
\end{array}\right) \frac{k-2 j}{(k+1) / 2+i-j} .
$$

Since $A_{(k-1) / 2, k}=0$, the equality (3.3) becomes

$$
u_{k^{n+1}}=\triangle^{(k-1) / 2} u_{k^{n}}^{k}-\sum_{i=0}^{(k-3) / 2} \triangle^{i} A_{i, k} u_{k^{n}}^{2 i+1}
$$

From (pp. 58, [3]), we have the combinatorial identity: for $1 \leq m \leq(k-3) / 2$

$$
\sum_{j=1}^{m}(-1)^{j} \frac{k-2 j}{k-m-j}\left(\begin{array}{c}
k \\
j
\end{array}\right)\left(\begin{array}{c}
k-m-j \\
m-j
\end{array}\right)=(-1)^{m} \frac{k}{k-m}\left(\begin{array}{c}
k-m \\
m
\end{array}\right) .
$$

If we replace $m$ by $\frac{k-1}{2}-i$ in (3.4), then we obtain $C_{i, k}=A_{i, k}$. Thus the proof is complete.

In a similar manner, we may give the following result:

Proposition 4. For $n>0$ and odd $k>1$,

$$
u_{k^{n+1}}=\Delta^{\frac{k-1}{2}} u_{k^{n}}^{k}-\sum_{i=0}^{\frac{k-3}{2}}(-1)^{\frac{k+1}{2}+i} \frac{2 k}{k-2 i-1}\left(\begin{array}{c}
\frac{k-1}{2}+i \\
2 i+1
\end{array}\right) \Delta^{i} u_{k^{n}}^{2 i+1} .
$$

Proof. For odd $k$, we get

$$
\begin{aligned}
u_{k^{n}}^{k} & =\frac{1}{\Delta^{k}} \sum_{j=0}^{k}\left(\begin{array}{l}
k \\
j
\end{array}\right)(-1)^{j} \beta^{j k^{n}} \alpha^{(k-j) k^{n}} \\
& =\frac{1}{\Delta^{\frac{k-1}{2}}}\left(u_{k^{n+1}}+\sum_{j=1}^{\frac{k-1}{2}}\left(\begin{array}{l}
k \\
j
\end{array}\right) u_{(k-2 j) k^{n}}\right) .
\end{aligned}
$$

So we write

$$
u_{k^{n+1}}=\Delta^{\frac{k-1}{2}} u_{k^{n}}^{k}-\sum_{j=1}^{\frac{k-1}{2}}\left(\begin{array}{l}
k \\
j
\end{array}\right) u_{(k-2 j) k^{n}}
$$

Using Lemma 1 and reversing the order of summation, we write

$$
\begin{aligned}
u_{k^{n+1}}= & \Delta^{\frac{k-1}{2}} u_{k^{n}}^{k}-u_{k^{n}} \sum_{j=1}^{\frac{k-1}{2}} \sum_{i=0}^{\frac{k-1}{2}-j}(-1)^{\frac{k-1}{2}+i-j} \\
& \times \frac{2(k-2 j)}{k-2 j+2 i+1}\left(\begin{array}{c}
k \\
j
\end{array}\right)\left(\begin{array}{c}
\frac{k-1}{2}+i-j+1 \\
2 i+1
\end{array}\right) \Delta^{i} u_{k^{n}}^{2 i}
\end{aligned}
$$




$$
\begin{aligned}
= & \Delta^{\frac{k-1}{2}} u_{k^{n}}^{k}-u_{k^{n}} \sum_{i=0}^{\frac{k-3}{2}} \sum_{j=1}^{\frac{k-1}{2}-i}(-1)^{\frac{k-1}{2}+i-j} \\
& \times \frac{2(k-2 j)}{k-2 j+2 i+1}\left(\begin{array}{c}
k \\
j
\end{array}\right)\left(\begin{array}{c}
\frac{k-1}{2}+i-j+1 \\
2 i+1
\end{array}\right) \Delta^{i} u_{k^{n}}^{2 i} .
\end{aligned}
$$

By simplifying, we derive

$$
u_{k^{n+1}}=\Delta^{\frac{k-1}{2}} u_{k^{n}}^{k}-u_{k^{n}} \sum_{i=0}^{\frac{k-3}{2}}(-1)^{\frac{k+1}{2}+i} \frac{2 k}{k-2 i-1}\left(\begin{array}{c}
\frac{k-1}{2}+i \\
2 i+1
\end{array}\right) \Delta^{i} u_{k^{n}}^{2 i}
$$

We give a recurrence relation for the sequence $\left\{v_{k^{n}}\right\}$ for odd $k$.

Proposition 5. For $n>0$ and odd $k>1$,

$$
v_{k^{n+1}}=v_{k^{n}}^{k}-\sum_{i=0}^{\frac{k-1}{2}} E_{i, k} v_{k^{n}}^{2 i+1},
$$

where

$$
E_{i, k}=\frac{2 k}{-k+2 i+1}\left(\begin{array}{c}
\frac{k-1}{2}+i \\
2 i+1
\end{array}\right)
$$

for $0 \leq i<(k-1) / 2$.

Proof. By the Binet formula of $\left\{v_{n}\right\}$ and the binomial expansion, we write

$$
\begin{aligned}
v_{k^{n}}^{k} & =\sum_{j=0}^{k}\left(\begin{array}{l}
k \\
j
\end{array}\right) \beta^{j k^{n}} \alpha^{(k-j) k^{n}} \\
& =v_{k^{n+1}}+\sum_{j=1}^{\frac{k-1}{2}}(-1)^{j}\left(\begin{array}{l}
k \\
j
\end{array}\right) v_{(k-2 j) k^{n}}
\end{aligned}
$$

By Lemma 1, we write

$$
v_{k^{n+1}}=v_{k^{n}}^{k}-\sum_{j=1}^{\frac{k-1}{2}} \sum_{i=0}^{\frac{k-1}{2}-j}(-1)^{j}\left(\begin{array}{c}
k \\
j
\end{array}\right)\left(\begin{array}{c}
\frac{k+1}{2}+i-j \\
2 i+1
\end{array}\right) \frac{k-2 j}{\frac{k+1}{2}+i-j} v_{k^{n}}^{2 i+1}
$$

and by reversing the order of summation, we get

$$
v_{k^{n+1}}=v_{k^{n}}^{k}-\sum_{i=0}^{\frac{k-1}{2}} \sum_{j=1}^{\frac{k-1}{2}-i}(-1)^{j}\left(\begin{array}{c}
k \\
j
\end{array}\right)\left(\begin{array}{c}
\frac{k+1}{2}+i-j \\
2 i+1
\end{array}\right) \frac{k-2 j}{\frac{k+1}{2}+i-j} v_{k^{n}}^{2 i+1}
$$


which, by the definition of $C_{i, k}$, gives

$$
v_{k^{n+1}}=v_{k^{n}}^{k}-\sum_{i=0}^{\frac{k-3}{2}} \sum_{j=1}^{\frac{k-1}{2}-i}(-1)^{j}\left(\begin{array}{l}
k \\
j
\end{array}\right)\left(\begin{array}{c}
\frac{k+1}{2}+i-j \\
2 i+1
\end{array}\right) \frac{k-2 j}{\frac{k+1}{2}+i-j} v_{k^{n}}^{2 i+1} .
$$

If we take $m=\frac{k-1}{2}-i$ in (3.4), we get

$$
v_{k^{n+1}}=v_{k^{n}}^{k}-\sum_{i=0}^{\frac{k-3}{2}} E_{i, k} v_{k^{n}}^{2 i+1},
$$

where

$$
E_{i, k}=\frac{2 k}{-k+2 i+1}\left(\begin{array}{c}
\frac{k-1}{2}+i \\
2 i+1
\end{array}\right) \text {. }
$$

For example, when $k=5$,

$$
v_{5^{n+1}}=v_{5^{n}}^{5}+5 v_{5^{n}}^{3}+5 v_{5^{n}}^{1} .
$$

We give a recurrence relation for the sequence $\left\{v_{k^{n}}\right\}$ for even $k$.

Proposition 6. For $n$ and even $k>0$,

$$
v_{k^{n+1}}=v_{k^{n}}^{k}-\sum_{i=0}^{\frac{k-2}{2}} H_{i, k} v_{k^{n}}^{2 i}
$$

where for $1 \leq m \leq k / 2$

$$
H_{i, k}=(-1)^{\left(\frac{k}{2}+i\right)}\left(\begin{array}{c}
\frac{k}{2}+i-1 \\
2 i
\end{array}\right) \frac{2 k}{-k+2 i} .
$$

Proof. It is easy to see that

$$
\begin{aligned}
v_{k^{n}}^{k} & =\sum_{j=0}^{k}\left(\begin{array}{l}
k \\
j
\end{array}\right) \beta^{j k^{n}} \alpha^{(k-j) k^{n}} \\
& =v_{k^{n+1}}-\left(\begin{array}{c}
k \\
\frac{k}{2}
\end{array}\right)+\sum_{j=1}^{\frac{k}{2}}\left(\begin{array}{l}
k \\
j
\end{array}\right) v_{(k-2 j) k^{n}},
\end{aligned}
$$

Then by Lemma 1 and reversing the order of summation, we get

$$
v_{k^{n}}^{k}=v_{k^{n+1}}-\left(\begin{array}{c}
k \\
\frac{k}{2}
\end{array}\right)+\sum_{j=1}^{\frac{k}{2}}\left(\begin{array}{l}
k \\
j
\end{array}\right) v_{(k-2 j) k^{n}}
$$




$$
\begin{aligned}
& =v_{k^{n+1}}+\left(\begin{array}{c}
k \\
\frac{k}{2}
\end{array}\right)+\sum_{j=1}^{\frac{k}{2}} \sum_{i=0}^{\frac{k}{2}-j}(-1)^{\left(\frac{k}{2}-j+i\right)}\left(\begin{array}{c}
k \\
j
\end{array}\right)\left(\begin{array}{c}
\frac{k}{2}-j+i \\
2 i
\end{array}\right) \frac{2\left(\frac{k}{2}-j\right)}{\frac{k}{2}-j+i} v_{k^{n}}^{2 i} \\
& =v_{k^{n+1}}+\sum_{i=0}^{\frac{k-2}{2}} \sum_{j=1}^{\frac{k}{2}-i}(-1)^{\left(\frac{k}{2}-j+i\right)}\left(\begin{array}{c}
k \\
j
\end{array}\right)\left(\begin{array}{c}
\frac{k}{2}-j+i \\
2 i
\end{array}\right) \frac{2\left(\frac{k}{2}-j\right)}{\frac{k}{2}-j+i} v_{k^{n}}^{2 i} \\
& =v_{k^{n+1}}+\sum_{i=0}^{\frac{k-2}{2}}(-1)^{\left(\frac{k}{2}+i\right)}\left(\begin{array}{c}
\frac{k}{2}+i-1 \\
2 i
\end{array}\right) \frac{2 k}{-k+2 i} v_{k^{n}}^{2 i} .
\end{aligned}
$$

Thus the proof is complete.

When $k=6$, we get

$$
v_{6^{n+1}}=v_{6^{n}}^{6}-6 v_{6^{n}}^{4}+9 v_{6^{n}}^{2}-2 v_{6^{n}}^{0} .
$$

Here we note that the coefficients of the formulas in (3.5) and (3.6) with adjusted sings appears to be the terms of the sequence A034807 in the OEIS.

\section{A Polynomial Representations for $v_{k^{n+1}}$}

In this section, we show that the generalized Lucas numbers $v_{k^{n+1}}$ are polynomials of the generalized Fibonacci numbers $u_{k^{n}}$ of degree $k$ for even $k$.

Proposition 7. For even $k>0$ and $n \geq 0$,

$$
v_{k^{n+1}}=\sum_{i=0}^{\frac{k-2}{2}} D_{i, k} \triangle^{i} u_{k^{n}}^{2 i},
$$

where $D_{i, k}$ is given by

$$
D_{i, k}=\frac{2 k}{k+2 i}\left(\begin{array}{c}
i+\frac{k}{2} \\
2 i
\end{array}\right)
$$

for $1 \leq m<k / 2$.

Proof. Consider

$$
\begin{aligned}
& u_{k^{n}}^{k}=\frac{1}{\triangle^{\frac{k}{2}}} \sum_{j=0}^{k}\left(\begin{array}{l}
k \\
j
\end{array}\right)(-1)^{j} \beta^{j k^{n}} \alpha^{(k-j) k^{n}} \\
& =\frac{1}{\Delta^{\frac{k}{2}}}\left((-1)^{\frac{k}{2}}\left(\begin{array}{c}
k \\
k / 2
\end{array}\right)+v_{k^{n+1}}-(-1)^{\frac{k}{2}}\left(\begin{array}{c}
k \\
k / 2
\end{array}\right) v_{0}\right. \\
& \left.+\sum_{j=1}^{\frac{k}{2}}(-1)^{j}\left(\begin{array}{l}
k \\
j
\end{array}\right) v_{(k-2 j) k^{n}}\right)
\end{aligned}
$$




$$
=\frac{1}{\triangle^{\frac{k}{2}}}\left(v_{k^{n+1}}-(-1)^{\frac{k}{2}}\left(\begin{array}{c}
k \\
k / 2
\end{array}\right)+\sum_{j=1}^{\frac{k}{2}}(-1)^{j}\left(\begin{array}{l}
k \\
j
\end{array}\right) v_{(k-2 j) k^{n}}\right)
$$

After using Lemma 1 and reversing the order of summation, we get for even $k$,

$$
\begin{aligned}
u_{k^{n}}^{k}= & \frac{1}{\triangle^{\frac{k}{2}}}\left(v_{k^{n+1}}+(-1)^{\frac{k}{2}}\left(\begin{array}{c}
k \\
k / 2
\end{array}\right)\right. \\
& \left.+\sum_{j=1}^{\frac{k-2}{2}} \sum_{i=0}^{\frac{k}{2}-j}\left((-1)^{j} \frac{2\left(\frac{k}{2}-j\right)}{\left(\frac{k}{2}-j\right)+i}\left(\begin{array}{c}
k \\
j
\end{array}\right)\left(\begin{array}{c}
\left(\frac{k}{2}-j\right)+i \\
2 i
\end{array}\right) \triangle^{i} u_{k^{n}}^{2 i}\right)\right)
\end{aligned}
$$

which becomes

$$
u_{k^{n}}^{k}=\frac{1}{\triangle^{\frac{k}{2}}}\left(v_{k^{n+1}}+\sum_{i=0}^{\frac{k-2}{2}} \sum_{j=1}^{\frac{k}{2}-i}\left((-1)^{j} \frac{2\left(\frac{k}{2}-j\right)}{\left(\frac{k}{2}-j\right)+i}\left(\begin{array}{c}
k \\
j
\end{array}\right)\left(\begin{array}{c}
\left(\frac{k}{2}-j\right)+i \\
2 i
\end{array}\right) \triangle^{i} u_{k^{n}}^{2 i}\right)\right) \text {. }
$$

If we take $m=\frac{k}{2}-i$ in (3.4) for $1 \leq m \leq k / 2$, the last equation takes the form:

$$
u_{k^{n}}^{k}=\frac{1}{\triangle^{\frac{k}{2}}}\left(v_{k^{n+1}}+\sum_{i=0}^{\frac{k-2}{2}} D_{i, k} \triangle^{i} u_{k^{n}}^{2 i}\right)
$$

where $D_{i, k}$ is the right hand side of (3.4) for $m=\frac{k}{2}-i$, that is,

$$
D_{i, k}=\left(\frac{-2 k}{k+2 i}\right)\left(\begin{array}{c}
i+\frac{1}{2} k \\
2 i
\end{array}\right) \text {. }
$$

Thus we have proved the conclusion.

When $k=6$, we have that

$$
v_{6^{n+1}}=125 u_{6^{n}}^{6}+150 u_{6^{n}}^{4}+45 u_{6^{n}}^{2}+2 .
$$

Note that the coefficients of the formula in (4.1) with adjusted sings appears to be the terms of the sequence A104064 in the OEIS.

\section{REFERENCES}

[1] P. Filipponi, "On the fibonacci numbers whose subscript is a power," Fibonacci Quart., vol. 34, no. 3, pp. 271-276, 1996.

[2] H. Prodinger, "On a sum of melham and its variants," Fibonacci Quart., vol. 46/47, no. 3, pp. 207-215, 2008/09.

[3] J. Riordan, Combinatorial identities. Huntington, N.Y.: Robert E. Krieger Publishing Co., 1979.

[4] A. Stinchcomb, "Problem b-769: First order cubic recurrence relation," Fibonacci Quart., vol. 32, no. 4, p. 373, 1994.

[5] M. N. S. Swamy, "On certain identities involving fibonacci and lucas numbers," Fibonacci Quart., vol. 35, no. 3, pp. 230-232, 1997. 
[6] D. C. Terr, "Solution b-769: The recurrence of $f_{3^{n}}$," Fibonacci Quart., vol. 33, no. 5, p. 468, 1995.

[7] Z. Usiskin, "Problem b-265: Fibonacci numbers from lucas products," Fibonacci Quart., vol. 11, no. 3, p. 333, 1973.

[8] Z. Usiskin, "Problem b-266: Lucas numbers from lucas products," Fibonacci Quart., vol. 11, no. 3, p. $333,1973$.

[9] S. Vajda, Fibonacci \& Lucas numbers, and the golden section, ser. Ellis Horwood Series: Mathematics and its Applications. New York: Ellis Horwood Ltd., Chichester; Halsted Press [John Wiley \& Sons, Inc.], 1989.

Authors' addresses

Emrah Kilic

TOBB Economics and Technology University, Mathematics Department, 06560 Sogutozu, Ankara Turkey

E-mail address: ekilic@etu.edu.tr

\section{Elif Tan}

Ankara University, Faculty of Sciences, Department of Mathematics, 06100 Tandogan, Ankara Turkey 